\title{
Hip Mobility and Flexibility for Track and Field Athletes
}

\author{
Joerg Teichmann1, Harald Burchardt'1, Rachel Tan1, Patrick Daniel Healy² \\ ${ }^{1}$ Rehamed Therapy Kuala Lumpur, Selangor, Malaysia \\ ${ }^{2}$ Tunku Abdul Rahman University College, Kuala Lumpur, Malaysia \\ Email: jorg@rehamedtherapy.com.my
}

How to cite this paper: Teichmann, J., Burchardt, H., Tan, R., \& Healy, P. D. (2021). Hip Mobility and Flexibility for Track and Field Athletes. Advances in Physical Education, 11, 221-231.

https://doi.org/10.4236/ape.2021.112017

Received: March 8, 2021

Accepted: May 8, 2021

Published: May 11, 2021

Copyright (c) 2021 by author(s) and Scientific Research Publishing Inc. This work is licensed under the Creative Commons Attribution International License (CC BY 4.0).

http://creativecommons.org/licenses/by/4.0/ (c) (i) Open Access

\begin{abstract}
Introduction: The objective of this paper is to demonstrate techniques on how to improve hip flexibility and mobility, especially for track and field athletes. It also seeks to explain the underlying importance of hip flexibility and mobility. When athlete's hips are stiff and inflexible, muscle imbalance may occur and increase the risk of injury. Muscle imbalance in the hip often causes knee and hip pain, due to the hip flexors and quadriceps overworking in order to compensate for weaker muscles. The hip mobility and flexibility exercises illustrated here are designed to significantly improve the range of motion (ROM) of the hips. Enhanced ROM can improve motor performance, skill execution, and even prevent injury. Excellent hip extension improves the ability to assist the hip in developing power. Methods: 9 Hip Mobility and 6 Hip Flexibility exercises are described in this paper. The exercises are to be performed $3 \times$ per week with at least 24 hours break in-between to achieve optimum results. Conclusion: This paper sought to outline the importance of hip mobility and flexibility, in particular for non-contact-sport athletes (such as those in track and field), and their effects on athletic performance and injury prevention. After proximately 6 weeks, the ROM of the hip will be expected to improve significantly.
\end{abstract}

\section{Keywords}

Hip Mobility and Flexibility, Track and Field, Injury Prevention, Range of Motion

\section{Introduction}

In general, sports injuries are more commonly associated with contact sports (such as hockey, rugby, football) as opposed to non-contact. However, research indicates that the opposite is in fact the case. Research conducted by (Lee et al., 
2017) and (Lee \& Lee, 2018) with the Perak SUKMA athletes revealed that of the 405 non-contact-sport athletes observed, 135 reported injuries, whereas of 533 contact-sport athletes only 105 claimed some form of injury. These results suggest that a higher proportion of injured athletes exist among non-contact sports, contrary to popular belief.

When an athlete's hips are shown to be tight or even immobile, muscle imbalance may occur and eventually lead to injury. Muscle imbalance in the hip often causes knee and hip pain, due to the hip flexors and quadriceps overworking in order to compensate for weaker, imbalanced muscles. Furthermore, the range of motion (ROM) of the hip would also be restricted, requiring the pelvis to posteriorly tilt to achieve the desired ROM, likely resulting in low back pain (Roach et al., 2015). Finally, the gluteal muscles should also be impinged in their function, the hamstrings would need to compensate, which may lead to hamstring injury. Performing hip flexibility and mobility exercises can often reduce the risk of these injuries from happening.

When it comes to flexibility and mobility exercises, stretching is commonly used to effect an increase in ROM (Behm et al., 2015). In addition, to improve techniques that are sports specific, the combination of passive and static-active stretching exercises has been shown to provide larger changes in ROM (Samson et al., 2012). However, contemporarily, dynamic stretching techniques are used more often by athletes as they can be sports specific, i.e., relating to common movements common in the sport. Dynamic stretching also increases body temperature and promotes blood circulation (Fletcher \& Jones, 2004), hence providing further benefit in the preparation for physical activity and/or sport.

Mobility is defined as the joint's ability to travel without any kind of constraint or pain, targeting the full ROM. In fact, the ability to move a joint through its complete ROM is arguably the most underrated aspect, a track and field athlete will explore when training to enhance their performance. It is a highly adaptable aspect of fitness and the benefits of mobility training are applicable at any age. Having mobile joints is important to maintain pain-free, unimpeded, fluid, and independent movement (Gummelt, 2015). Therefore, it can be inferred that a key component to avoiding injuries is improving ROM.

Mobility training is the process of improving mobility in all joints. Overall, having a healthy ROM in all the joints of your body means your body is free to move and adjust to its position in the most efficient way (Ilano, 2020). Mobility exercises work to reduce the potential imbalances, the body develops over time, ultimately helping to diminish the risk of injury. Additionally, mobility exercises can stimulate and circulate the synovial fluid in the bursa, which effectively "cleans" the joint. This is important as the joint has no direct blood supply and is nourished by this synovial fluid, which also simultaneously removes waste products.

Flexibility is defined as a muscle's ability to lengthen (Kassel, 2018). Flexibility also refers to the capacity of a connective tissue to elongate temporarily. Typically, flexibility requires external force to reach the desired positions, for 
example, a foreign body or stretching device affecting a particular body part while the athlete is stationary.

Flexibility training, particularly among athletes who train for high performance, works to reduce soreness and stiffness (Gummelt, 2015). It is also a form of relaxation that can not only have a positive effect on physical health and performance, but also mental fitness (Gummelt, 2015). For athletes to achieve peak performance, full length of the muscle must be utilized, as the muscles may not be able to provide the explosiveness required for a specific movement if the muscles are not flexible enough (Hunter \& Marshall, 2002). For instance, tight hip flexors tend to shorten the ROM of a full step while in sprint, inhibiting performance.

\section{Injuries That Come Along with Restricted Hip ROM}

The muscular system of the human body is all connected meaning the stability of one area can directly influence that of another. The reason for this chosen topic is to give advice for track and field athletes on hip mobility and flexibility, but in order to do that, it is crucial to bring awareness of common injuries that come along with restricted hip ROM. There are instances where the blame gets put on the running technique and the type of shoes for lack of performance or pain, but it is important to understand that these issues could be potentially caused by problems further up the kinetic chain.

Studies have been conducted throughout the years looking at the correlation between low back pain and restricted hip ROM. A study conducted by Roach et al. (2015) looked at hip extension mobility evaluating two groups, those with and without low back pain. The results showed that those with low back pain had -4.16 degrees hip extensions and those without low back pain had 6.78 degrees hip extension (Roach et al., 2015).

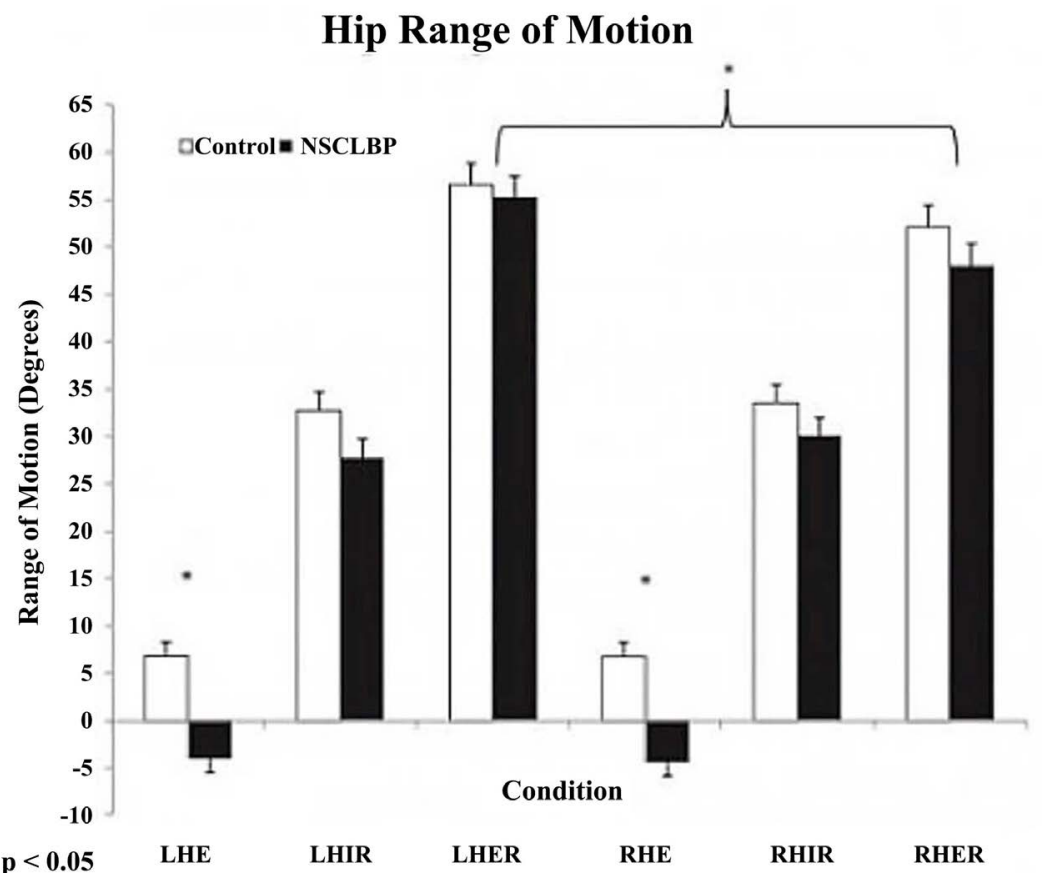


The results shown in the bar chart above highlight those with low back pain have a negative degree of hip extension whereby they won't be able to extend to a neutral position. The human body tends to compensate when one limb doesn't work properly, hence it extends the spine more which leads to more lower back pain (Roach et al., 2015). This compensatory pattern could potentially lead to overuse, fatigue and altered motor activation patterns in the hip and lumbar spine leading to low back pain and injury. Limitations of the hip have been suggested to be present in some conditions of lumbar spine problems, as well as in more distal lower extremity pathologies (Reiman et al., 2009: p. 18). Hip mobility limitations have been found in individuals with osteoarthritis, any groin pain that relates to sports (Taylor et al., 2011: p. 14), and hip labral tear (Agricola et al., 2012).

A study conducted by Mills (Mills, 2014), investigated the association between hip flexor tightness and reduced gluteus maximus activation during an overhead squat. The study had 20 female college soccer players with hip flexor tightness and another 20 matched controls with 15 degrees or below horizontal. Above horizontal on a modified Thomas Test was used in this study for the group with tight hip flexors. Results showed that gluteus maximus activation was significantly lower in the group with tight hips than the control group. The gluteus maximus to bicep femoris coactivation ratio was also significantly lower in the group with tight hip flexors (Mills, 2014).

In running gait, we need this triple extension to be driven top-down from the hip, with gluteus maximus and the hamstrings contracting powerfully to drive the femur backwards from the hip (Dunne, 2021). For this hip extension pattern to be most effective, we need stability and control around the pelvic region, to provide a stable base (Dunne, 2021). Athletes would need to work on the quality of movement coming from their hips during a running gait.

When you run, the pelvic region acts as a crossroads for forces working through the body. Its articulations with the sacrum and thus lumbar spine dictate much of the position and movement of the trunk during running gait. The pelvis serves as the root and insertion point for a number of essential muscles that are involved in both movement and stability (Dunne, 2021).

There are various methods to self-mobilize for individuals with hip intra- or extra-articular pathology. The goal is to improve capsule and connective tissue mobility, as well as improve muscle re-education. Additionally, often with these techniques the recruited muscles can assist with optimizing hip joint movement. This improved joint movement may significantly alleviate the athlete or client's symptoms (Reiman \& Matheson, 2013). With proper in-clinic instructions, individuals may be able to enhance their treatment plan by performing a series of selected self-mobilization techniques and end range exercises as part of a home rehabilitation program. Application of these techniques is beneficial after the clinician has performed them directly on the client in the clinic (Reiman \& Matheson, 2013). 


\section{Hip Mobility and Flexibility Program}

\section{Description}

Table 1 describes 9 mobility exercises specifically for improving hip mobility. As shown in the picture for the first exercise, the athlete is in a deep sumo squat position targeting the glutes and hip adductors, adductor brevis, longus and magnus to be more specific (Halfman, 2012). However, the athlete is bouncing in that specific position from left to right as a progressed way of doing this exercise; a regressed way is that the athlete should stay in that position.

Moving on to exercise 2, this exercise targets the adductors. As shown in the picture, the athlete has one leg kneeling on the ground while the other, working leg is straight to the side of the body. This exercise helps with improving adductor length and mobility, which is targeted at improving hip adduction ROM. Exercise 3 is a progression of Exercise 2. Instead of working on each leg individually, it stretches both adductors at the same time.

Exercise 4 is used to improve greater mobility of the spine, a progressed version of which is performed in exercise 5 . In every movement the body does, the trunk muscles play an important role. They support walking, maintain equilibrium, and provide stability for the whole body. Rotation exercises like in 4 and 5 can help enhance trunk stability and strength, which will improve general health and increase sports performance (Leal, 2020).

Exercise 6 depicts a progressed version of the 90/90 hip stretch. This exercise targets the deepest layers of tissue associated with the joint, such as the joint capsule. The constant change of position puts more work in than just sitting in the 90/90 hip stretch position (Hanrahan, 2017). This is important as a restricted capsule has a detrimental effect on the body's overall flexibility (Hanrahan, 2017).

Exercises 7 - 9 are related but target the joint in different angles: these are so-called "banded joint distraction exercises" (for the hip). The joint distraction exercises use a resistance band, which acts as a wedge to separate the joint surfaces from one another (Guillot et al., 2019). The separation of the articulating surfaces will provide more space for synovial fluid to fill the joint, reducing friction and providing the body a more fluid motion (Guillot et al., 2019).

\section{Description}

Looking at Table 2, the first exercise is a regressed version of the wide-legged forward bend. It utilises the walking motion by the hands as an external force to passively stretch the lower body, targeting the adductors and even the hamstrings.

Exercise 2 is commonly known as the knee-to-chest stretch and is typically used to release tension on the lower back. This helps reduce stiffness associated with spinal arthritis and spinal stenosis (Asher, 2020), which is important for athletes of all sports. Furthermore, it promotes spinal flexion by enabling the natural chain reaction, from the thigh to the hip to the lower back, to occur (Asher, 2020).

Exercise 3 is a progressed version of the $90 / 90$ hip stretch, and is similar to mobility exercise 6 but as more of a passive stretch (Spina, 2020). Staying in the position shown, the exercise stretches the gluteus minimus muscle and is a good indicator of hip health (Spina, 2020). 
Table 1. Mobility exercises.

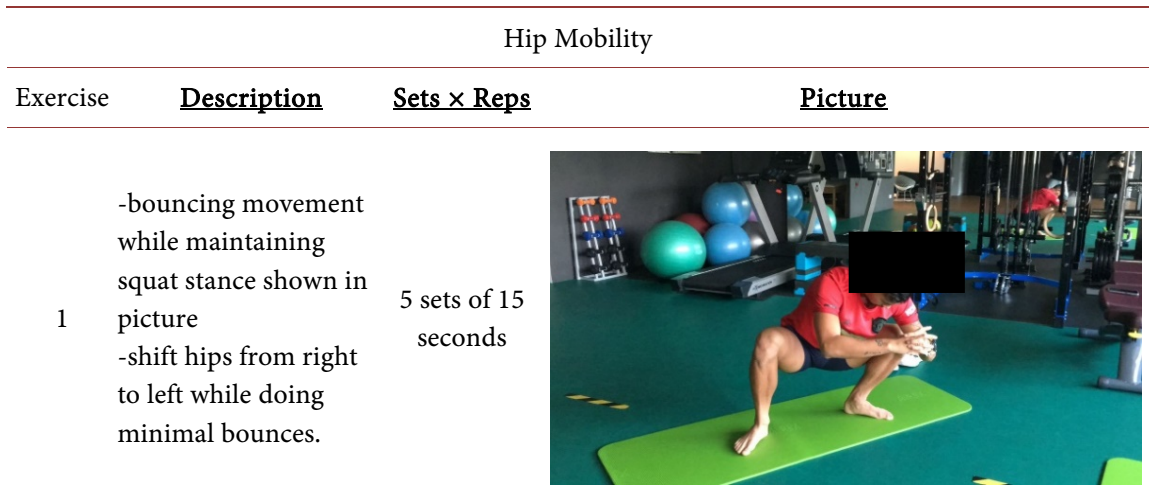

-Maintain position shown in picture. -move hips front an back while maintaining body in straight position

-maintain position shown in picture. -legs spread wide, knees fully extended. -move hips up and down while keeping the back straight

-lay down, arms lay out above head level -knees up, flexed (bent)

-keep feet together then move knees left and right

-keep shoulders on the ground.

-rotate hips.

5 sets of 15

seconds

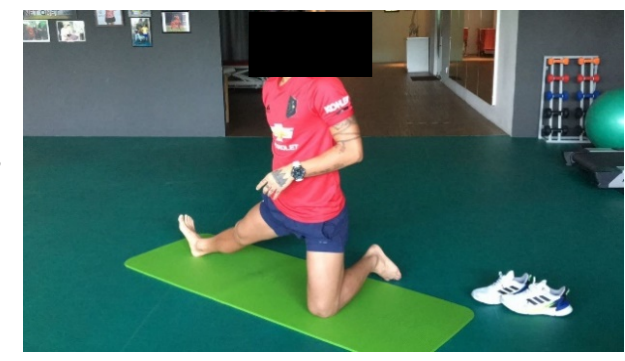

5 sets of 15 seconds
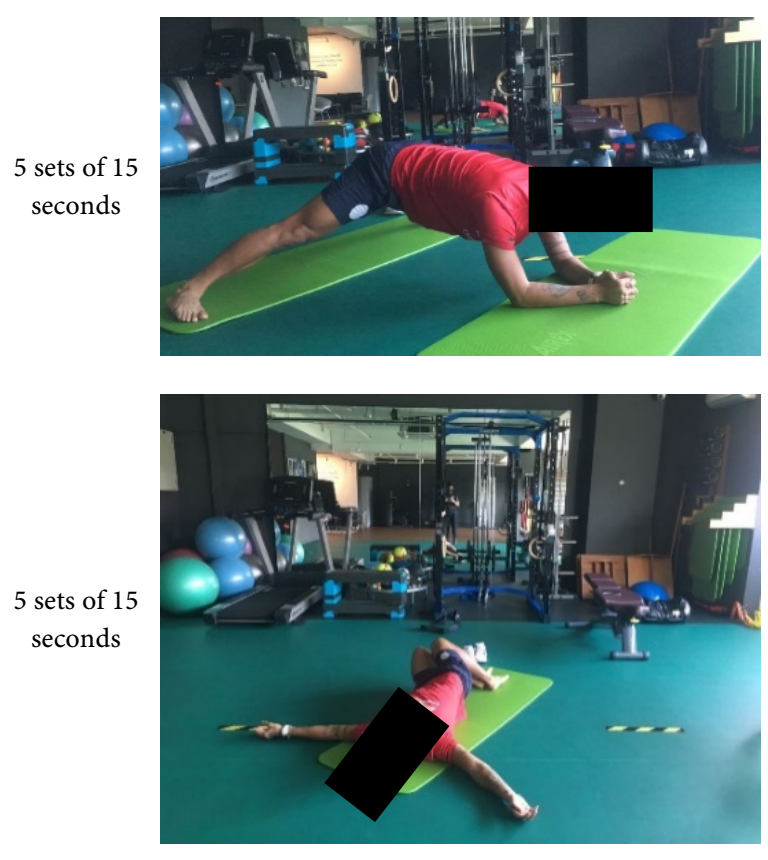

-lay down flat. -arms open wide. -move one leg to opposite hand as shown in picture.

5 -repeat for other side. -both knees have to be straight.

-hold for 15 seconds then move to the opposite direct
5 sets of 15 seconds

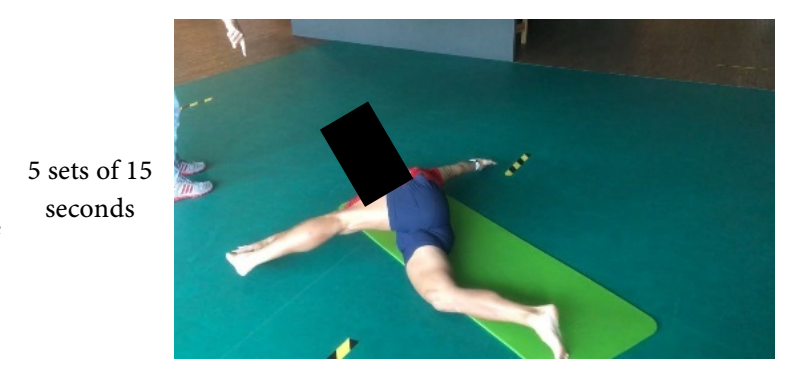




\section{Continued}

-start in position shown in the first picture.

-as low as possible to the ground (touching if possible)

-extend both knees, and slowly move to the opposite of the first position (shown in the last picture)

-repeat the steps.
Maintain in lunge position while the resistance band pulls back the hip of the back leg.

-move the hips back and forward

throughout the set.

-maintain in lunge position while the resistance band pulls the hip of the front leg to adduction.

-resist and maintain a proper straight lunge position.

-maintain in lunge position while the resistance band pushes 5 sets of 15 the hip forward. -move the hips back and forward throughout the set.

5 sets of 15 side side
5 sets of 15 each side
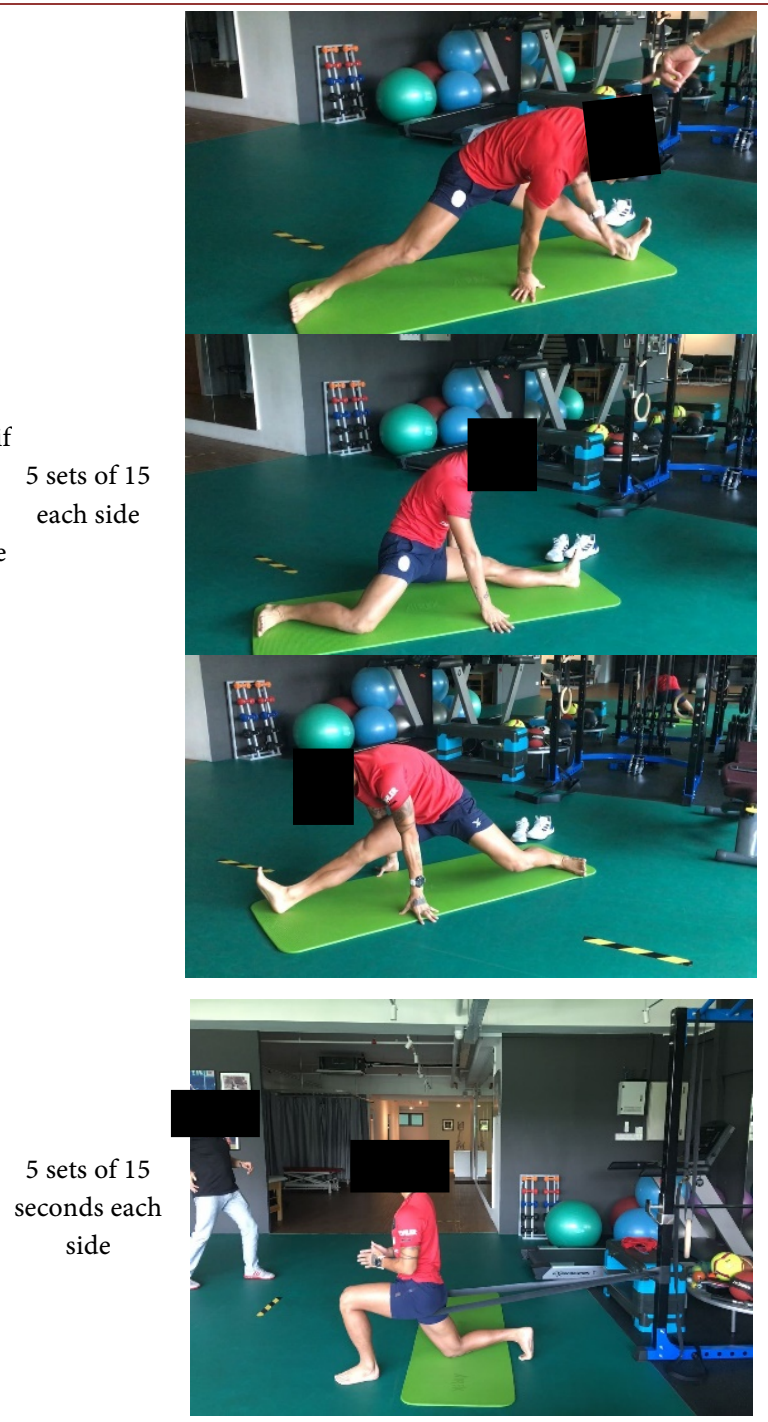
seconds each seconds each
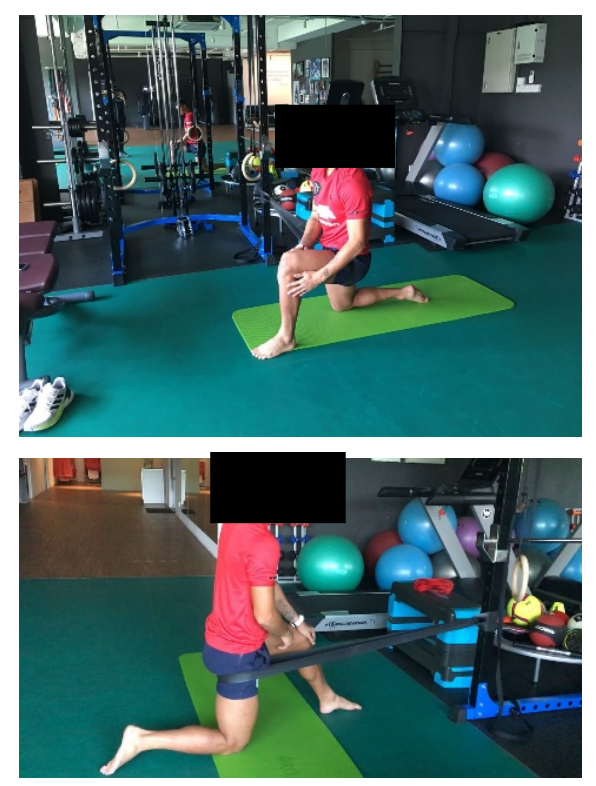
Table 2. Flexibility exercises.

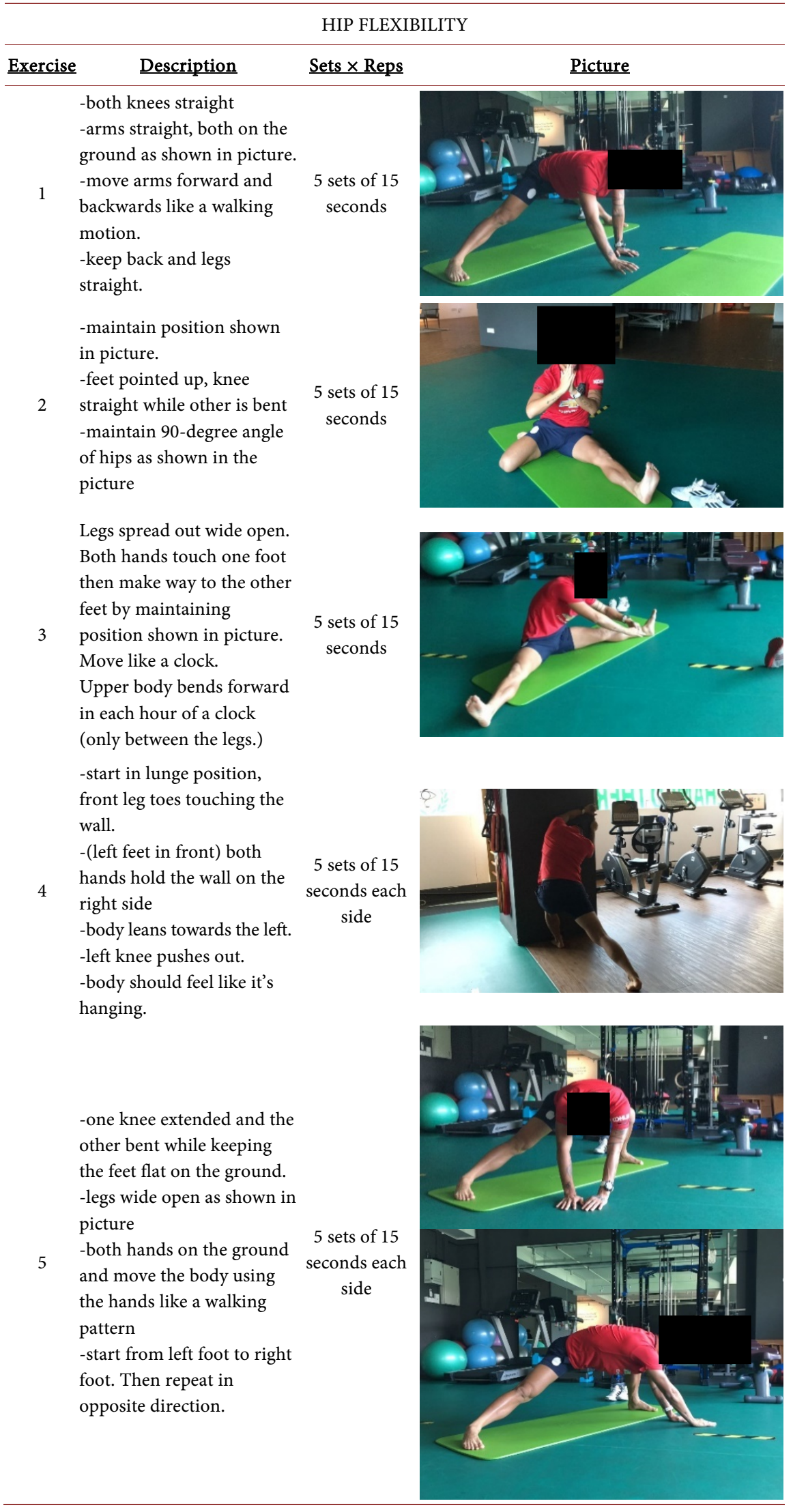




\section{Continued}

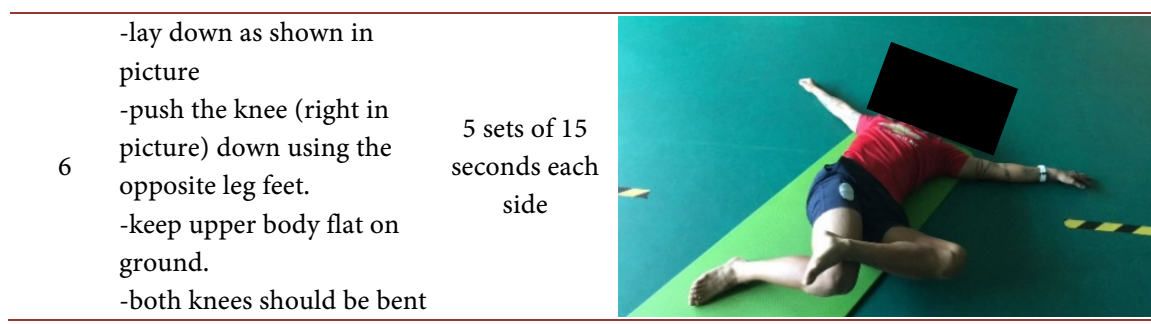

A progressed version of the wide angle seated forward bend pose is depicted in exercise 4 , in which the upper body is pointed forward and rotates like a clock (Watts, 2020). This way the upper body tends to reach out in different directions, focusing on stretching the hamstrings and groin muscles (Watts, 2020).

Exercise 5 is known as the wall stretch. Holding this position targets the upper and lower Quadratus Lumborum (Mark, 2020). This exercise relies on the upper arms to take the weight of the body, hanging the body by the arms. The middle part of the body is bent while the legs act as anchors, using the bodyweight to sink into the stretch (Mark, 2020).

In the second last exercise, the athlete stays in a side lunge position stretching the groin, adductors and even the gracilis. However, this specific exercise is regressed, using the upper body to act as a front support as the athlete bends over. Moving in a walking motion using the hands helps increase the ROM in various directions.

Lastly, exercise 7 is commonly known as the external hip rotator stretch but with additional support from the opposite leg. The support acts as an external force to depress the knee using the foot of the other leg. This results in a stretch down the iliotibial (IT) band, which is located at the side of the outer thigh.

\section{Conclusion}

This paper sought to outline the importance of hip mobility and flexibility, in particular, for non-contact-sport athletes (such as those in track and field), and their effects on athletic performance and injury prevention. In total 15 exercises were illustrated and described, with the goal of improving hip ROM and flexibility.

The exercises above can be performed either a few hours before any kind of training or in the late evenings, in both cases achieving similar improvements in ROM. However, each has its own benefit. Stretching in the morning helps relieve any tension or pain from sleeping the night before. It can also increase blood flow, preparing your body for the day ahead and sports training. Stretching before bed can help relax the muscles and prevent pain or soreness in the mornings.

The regime of " 5 sets of 15 seconds" was prescribed to habituate the muscle spindles and reduce signaling as a result of the prolonged stretching periods. Gradually it will train the stretch receptors to allow the muscles to lengthen 
more. Holding in the positions for the recommended time and sets will also allow the lengthening reaction to happen, helping those muscles that are stretched to relax.

To achieve optimal results, it is recommended to perform this program at least 3 times a week, with at least 24 hours break in-between each time. After at least 6 weeks, the ROM of the hip will be expected to improve significantly. Furthermore, other results can be seen in better/longer strides while running, and even kicking more comfortably resulting from longer swing time. Finally, reduced muscle imbalance allows the body to utilize more of the correct muscles, instead of strong muscles compensating the weak muscles, which as mentioned above can lead to injury as the muscle becomes overused.

\section{Conflicts of Interest}

The authors declare no conflicts of interest regarding the publication of this paper.

\section{References}

Agricola, R., Heijboer, M. P., Bierma-Zeinstra, S. M. A., Verhaar, J. A. N., Weinans, H., \& Waarsing, J. H. (2012). Cam Impingement Causes Osteoarthritis of the Hip: A Nationwide Prospective Cohort Study. Annals of the Rheumatic Diseases, 72, No. 6. https://doi.org/10.1136/annrheumdis-2012-201643

Asher, A. (2020). Knees to Chest Stretch for Low Back Muscles. https://www.verywellhealth.com/knees-to-chest-exercise-296870

Behm, D. G., Blazevich, A. J., Kay, A. D., \& McHugh, M. (2015). Acute Effects of Muscle Stretching on Physical Performance, Range of Motion, and Injury Incidence in Healthy Active Individuals. Applied Physiology, Nutrition, and Metabolism, 41, 1-11. https://doi.org/10.1139/apnm-2015-0235

Dunne, J. (2021). How Does Hip Extension Affect Your Running Technique? https://www.kinetic-revolution.com/running-its-all-in-the-hips/

Fletcher, I. M., \& Jones, B. (2004). The Effects of Different Warm up Stretch Protocols on 20 Meter Sprint Performance in Trained Rugby Union Players. Journal of Strength and Conditioning Research, 18, 885-888. https://doi.org/10.1519/00124278-200411000-00035

Guillot, A., Kerautret, Y., Queyrel, F., Schobb, W., \& Di Rienzo, F. (2019). Foam Rolling and Joint Distraction with Elastic Band Training Performed for 5-7 Weeks Respectively Improve Lower Limb Flexibility. Journal of Sports Science \& Medicine, 18, 160-171.

Gummelt, D. (2015). The Impact of Flexibility Training on Performance. https://www.acefitness.org/education-and-resources/professional/expert-articles/5598/t he-impact-of-flexibility-training-on-performance/

Halfman, P. (2012). Sumo Squat Stretch. http://www.tennis-conditioning.com/2012/08/sumo-squat-stretch/

Hanrahan, J. (2017). The best Hip Mobility Exercise. Period. https://jackhanrahanfitness.com/best-hip-mobility-exercise-period/

Hunter, J. P., \& Marshall, R. N. (2002). Effects of Power and Flexibility Training on Vertical Jump Technique. Medicine \& Science in Sports \& Exercise, 34, 478-486. https://doi.org/10.1097/00005768-200203000-00015 
Ilano, J. (2020). Joint Mobility Exercises for Health, Function, and Workout Preparation. Seattle, Washington: GMB Fitness.

Kassel, G. (2018). What's The Difference between Mobility vs. Flexibility? https://barbend.com/mobility-vs-flexibility/

Leal, D. (2020). How to Perform Trunk Rotation. https://www.verywellfit.com/how-to-perform-trunk-rotation-techniques-benefits-varia tions-4690852

Lee, A. C., \& Lee, Z. C. (2018). Injury Profile of Contact-Sports for Perak Athletes in Malaysia. International Journal of Academic Research in Business \& Social Science, 8, 965-976. https://doi.org/10.6007/IJARBSS/v8-i9/4672

Lee, A. C., Sankaravel, M., Mondam, S., \& Kuang, P. F. (2017). Injury Profile of Non-Contact Sports for Perak SUKMA Athletes. Journal of Fundamental and Applied Sciences, 9, 1141. https://doi.org/10.4314/jfas.v9i6s.84

Mark (2020). Quadratus Lumborum Stretch. https://posturedirect.com/quadratus-lumborum-stretch/

Mills (2014). Effect of Limited Hip Flexor Length on Gluteal Activation during an Overhead Squat in Female Soccer Players. Journal of Athletic Training, 49, S-83.

Reiman, M. P., \& Matheson, J. W. (2013). Restricted Hip Mobility: Clinical Suggestions for Self-Mobilization and Muscle Re-Education. International Journal of Sports Physical Therapy, 8, 729-740.

Reiman, M. P., Bolgla, L. A., \& Lorenz, D. (2009). Hip Functions Influence on Knee Dysfunction: A Proximal Link to a Distal Problem. Journal of Sport Rehabilitation, 18, 33-46. https://doi.org/10.1123/jsr.18.1.33

Roach, S. M., Juan, J. G. S., Suprak, D. N., Lyda, M., Bies, A. J., \& Boydston, C. R. (2015). Passive Hip Range of Motion Is Reduced in Active Subjects with Chronic Low Back Pain Compared to Controls. International Journal of Sports Physical Therapy, 10, 13-20.

Samson, M., Button, D. C., Chaouachi, A., \& Behm, D. G. (2012). Effects of Dynamic and Static Stretching within General and Activity Specific Warm up Protocols. Journal of Sports Science and Medicine, 11, 279-285.

Spina, A. (2020). 3 Hip Mobility Exercises \& Why You Should Do Them. https://www.onnit.com/academy/increase-hip-mobility/

Taylor, C. J., Pizzari, T., Ames, N., Orchard, J. W., Gabbe, B. J., \& Cook, J. L. (2011). Groin Pain and Hip Range of Motion Is Different in Indigenous Compared to Non-Indigenous Young Australian Football Players. Journal of Science and Medicine in Sport, 14, 283-286. https://doi.org/10.1016/j.jsams.2011.02.002

Watts, M. (2020). Upavistha Konasana. https://www.siddhiyoga.com/upavistha-konasana-wide-angle-seated-forward-bend-pose 